\title{
The metallicity distribution of the halo and the satellites of the Milky Way in the hierarchical merging paradigm
}

\begin{abstract}
N. Prantzos
Institut d'Astrophysique de Paris, UMR7095 CNRS, Univ. P. \& M. Curie, 98bis Bd. Arago, 75104 Paris, France

e-mail: prantzos@iap.fr

Received 26 December 2007 / Accepted 28 June 2008

ABSTRACT

Aims. To account for the observed differential metallicity distribution (DMD) of the Milky Way halo, a semi-analytical model is presented in the framework of the hierarchical merging paradigm for structure formation.

Methods. It is assumed that the Milky Way halo is composed of a number of sub-haloes with properties either as observed in the dwarf satellite galaxies of the Local group (shape of metallicity distribution, effective yield) or derived from calculations of structure formation (sub-halo distribution function).

Results. With reasonable assumptions for the parameters involved, we find that the overall shape and effective yield of the Galactic halo DMD can be reproduced in the framework of such a simple model. The low metallicity tail of the DMD presents a deficiency of stars with respect to the simple model predictions (akin to the G-dwarf problem in the solar neighborhood); it is suggested that an early infall phase can account for that problem, as well as for the observed DMDs of dwarf satellite galaxies.

Conclusions. Accretion of galaxies similar (but not identical) to the progenitors of present day dwarf satellites of the Milky Way may well have formed the Galactic halo.
\end{abstract}

Key words. Galaxy: abundances - Galaxy: formation - Galaxy: halo

\section{Introduction}

The metallicity distribution of long-lived stars is one of the most powerful probes of galactic chemical evolution. Indeed, the shape of the metallicity distribution is independent of the rate at which stars are formed. It depends essentially on the stellar initial mass function (IMF), which fixes the "intrinsic" (true) yield of the stellar population and the loss or gain of (gaseous) mass from the galaxian system (outflow or inflow, see next section and Prantzos 2007a). In the case of the solar neighborhood, for instance, the shape of the metallicity distribution suggests that the local disk was constructed by infall on long timescales (e.g. Boissier and Prantzos 1999).

The regular shape of the metallicity distribution of the Milky Way (MW) halo can readily be explained by the simple model of galactic chemical evolution with outflow, as suggested by Hartwick (1976, see also Sect. 2.1). However, that explanation lies within the framework of the monolithic collapse scenario for the formation of the MW (Eggen et al. 1962). It seems dificult to keep that interpretation in the currently dominant paradigm of hierarchical galaxy formation. A coherent interpretation should be sought in the framework of the new paradigm according to which the MW halo was formed from the hierarchical merging of smaller components (sub-haloes).

Several attempts to account for the metallicity distribution of the MW halo in the modern framework were undertaken in recent years, starting with the work of Bekki \& Chiba (2001). They model a galaxy closely resembling the MW and despite the low spatial resolution of their simulations $(2 \mathrm{kpc})$, they are able to provide a halo metallicity distribution with a peak at the observed metallicity $([\mathrm{Fe} / \mathrm{H}]=-1.6)$, but with a shape that is much narrower than the observed one; they obtain a double peaked metallicity distribution (which is not observed), probably reflecting late major mergers due to the low mass resolution. In their five MW-type simulations (in a cosmological framework and using various prescriptions for ouflow/feedback), Scanapieco \& Broadhurst (2001) find no resemblance of the resulting halo metallicity distributions with the observed one. This is also the case in Font et al. (2006), who find systematically too metalrich haloes with respect to the MW halo (see their Fig. 6). On the contrary, using semi-analytical models (starting with the extended Press-Schecter formalism and using various prescriptions for star formation, feedback and mixing of the stellar ejecta), Tumlinson (2006) and Salvadori et al. (2007) find rather good agreement between the model and observed halo metallicity distribution.

Independently of their success or failure in reproducing the observations, those recent models provide little or no physical insight into the physical processes that shaped the metallicity distribution of the MW halo. Indeed, if the halo was built from a large number of sucessive mergers of sub-haloes, why is its metallicity distribution so well described by the simple model with outflow (which refers to a single system)? And what determines the peak of the metallicity distribution at $[\mathrm{Fe} / \mathrm{H}]=\sim-1.6$, which is (successfully) interpreted in the simple model by a single parameter (the outflow rate)? Even in the succesful models cited in the previous paragraph, no clear answer to these questions is provided.

In this work we present an attempt to built the halo metallicity distribution analytically, in the framework of the hierarchical merging paradigm. Preliminary results have been presented in Prantzos (2007b). It is assumed that the building blocks were galaxies with properties similar (but not identical) to those of the Local group dwarf galaxies that we observe today. In that way, 
the physics of the whole process may become more clear. The plan of the paper is as follows: in Sect. 2 we present the simple model of the outflow and its overall successful description of observations; we discuss, in particular, some issues related to its low- and high- metallicity tails. In Sect. 3 we present the properties of the sub-haloes that formed the MW halo. These are derived either from observations of the Local Group dwarf galaxies (the shape of the metallicity distribution, Sect. 3.1 and the effective yield, Sect. 3.2) or from the theory of large scale structure formation (the distribution function of baryonic sub-haloes, Sect. 3.3). The results of the model are presented in Sect. 3.4 and summarized in Sect. 4.

\section{The halo metallicity distribution and the simple model}

The halo metallicity distribution for field stars was well established in the metallicity range $-2.2<[\mathrm{Fe} / \mathrm{H}]<-0.8$ by two major surveys in the 1990s (Ryan \& Norris 1991; Carney et al. 1996). An ongoing survey, known as the Hamburg/ESO survey, seeks to establish the form of the metallicity distribution at even lower metallicities. Its results should be considered as representative down to $[\mathrm{Fe} / \mathrm{H}] \sim-3$, and thus they supercede the survey of Ryan \& Norris (1991) below $[\mathrm{Fe} / \mathrm{H}]=-2$. For $[\mathrm{Fe} / \mathrm{H}]>-2.2$, various biases affect the results of the Hamburg/ESO survey. Figure 1 displays a composite of the Ryan \& Norris (1991) and the Hamburg/ESO surveys, adjusted to fit each other at $[\mathrm{Fe} / \mathrm{H}]=-2.2$. In this work we shall consider the main body of that distribution, and we shall only briefly discuss some issues related to its low- and high-metallicity tails.

As already noticed a long time ago (Hartwick 1976) the halo metallicity distribution is closely described by the simple model of galactic chemical evolution (GCE). In the framework of that model, the metallicity $Z$ is given as a function of the gas fraction $\mu$ as

$Z=p \ln \left(\frac{1}{\mu}\right)+Z_{0}$

where $Z_{0}$ is the initial metallicity of the system (zero for all isotopes, except those created in the Big Bang) and $p$ is the yield of a stellar generation, i.e. the newly created mass of a metal returned to the ISM per unit mass blocked in stars (both long lived stars and stellar remnants). Metallicities and yield are expressed in units of the solar metallicity $Z_{\odot}$. The yield depends on the stellar nucleosynthesis models and on the adopted stellar initial mass function (IMF). If the system evolves at a constant mass (closed box), the yield is called the true yield, otherwise (i.e. in case of mass loss or gain) it is called the effective yield; its value is then always lower than the one of the true yield and it can be analytically evaluated in some specific cases (see e.g. Edmunds 1990).

Taking into account that the number of stars is proportional to the stellar mass (for a constant IMF) and that the star mass fraction $\mu_{*}$ is given by $\mu_{*}=1$. $-\mu$, one may use Eq. (1) to derive the differential metallicity distribution (DMD) of the system as:

$\frac{\mathrm{d}\left(n / n_{1}\right)}{\mathrm{d}(\log Z)}=\frac{\ln 10}{1-\exp \left(-\frac{Z_{1}-Z_{0}}{p}\right)} \frac{Z-Z_{0}}{p} \exp \left(-\frac{Z-Z_{0}}{p}\right)$

where $Z_{1}$ is the final metallicity of the system and $n_{1}$ the total number of stars (having metallicities $\leq Z_{1}$ ). This function has a maximum for $Z-Z_{0}=p$, allowing one to evaluate easily the effective yield $p$ if the DMD is determined observationally.

The DMD of field halo stars peaks at $[\mathrm{Fe} / \mathrm{H}] \sim-1.6$, suggesting an effective halo yield $p_{\text {Halo }} \sim 1 / 40 Z_{\odot}$ for Fe. This is to be compared to the true yield obtained in the solar neighborhood. The peak of the local DMD is located in the range $-0.2<[\mathrm{Fe} / \mathrm{H}]<0$, depending on the adopted metallicity calibration (see e.g. Haywood 2006; Holmberg et al. 2007). Assuming that the peak is at $[\mathrm{Fe} / \mathrm{H}]=-0.1$, it points to a local yield of $p_{\text {Disk }}=0.8 Z_{\odot}$. This value is not very different from the yield derived for the bulge of the MW, which evolved more or less as a closed box (as suggested from its metallicity distribution, see e.g. Fulbright et al. 2006); this is considered to be the true yield (assuming the same IMF between local disk and the bulge). Taking into account that $\sim 60-65 \%$ of solar Fe are produced by SNIa and only $\sim 35-40 \%$ by SNII (e.g. Goswami \& Prantzos 2000) it turns out that the true Fe yield of SNII during the local disk evolution was $p_{\text {Disk }} \sim 0.32 Z_{\odot}$ and, consequently, the effective Fe yield of SNII during the halo phase (where they dominated $\mathrm{Fe}$ production) was $p_{\text {Halo }} \sim 0.08 p_{\text {Disk }}$.

The simplest interpretation of such a difference between the effective yields in the halo and the local disk still remains the one of Hartwick (1976), who suggested that outflow at a rate $F=k \Psi$ (where $\Psi$ is the star formation rate or SFR) occured during the halo formation. In the framework of the simple model of GCE such outflow reduces the true yield $p_{\text {True }}$ to its effective value $p_{\text {Halo }}=p_{\text {True }}(1-R) /(1+k-R)($ e.g. Pagel 1997) where $R$ is the return mass fraction $(R \sim 0.32-0.36$ for most of the modern IMFs, e.g. the one of Kroupa et al. 1993 or of Kroupa 2002). The halo data suggest then that the ratio of the outflow to the star formation rate was

$k=(1-R)\left(\frac{p_{\text {True }}}{p_{\text {Halo }}}-1\right)$

and, by replacing $p_{\text {True }}$ with $p_{\text {Disk }}$ one finds that $k \sim 7-8$ times the star formation rate. This high outflow rate could be interpreted either as gas expulsion after heating by supernova explosions, or as simple flowing of gas through the system, e.g. towards the Galaxy's bulge.

Although the simple model of GCE with outflow fits extremely well the bulk of the halo DMD, the situation is less satisfactory for the low metallicity tail of that DMD. The problem (a lack of low metallicity stars w.r.t. the predictions of the simple model) was noticed sometime ago and prompted some interesting scenarios concerning the physics of the early galaxy, e.g. pre-enrichment by a putative population of Pop. III stars (Norris 1999) or various possibilities of inhomogeneous early evolution (Tsujimoto et al. 1999; Oey 2003). Prantzos (2003) showed that the situation is in fact even worse if the instantaneous recycling approximation (adopted in all previous works on the problem) is dropped and the finite lifetimes of stars are taken into account. Noting that the situation is reminiscent of the G-dwarf problem in the solar neighborhood, he further suggested that a similar solution should also apply, namely an early phase of rapid infall (on a time scale of less than $0.1 \mathrm{Gyr}$ ) forming the Milky Way's halo. Such a scenario is illustrated in Fig. 1. It may account for the "G-dwarf" problem of the halo, at the price of introducing more degrees of freedom than the simple model. At present, the situation is not clear regarding the low metallicity tail of the halo DMD. We shall present some tentative conclusions, based on preliminary data, in Sect. 3.5; however, more sound conclusions should be drawn only after the definitive results of the full Hamburg/ESO survey (Schörk et al., in preparation) become available. 


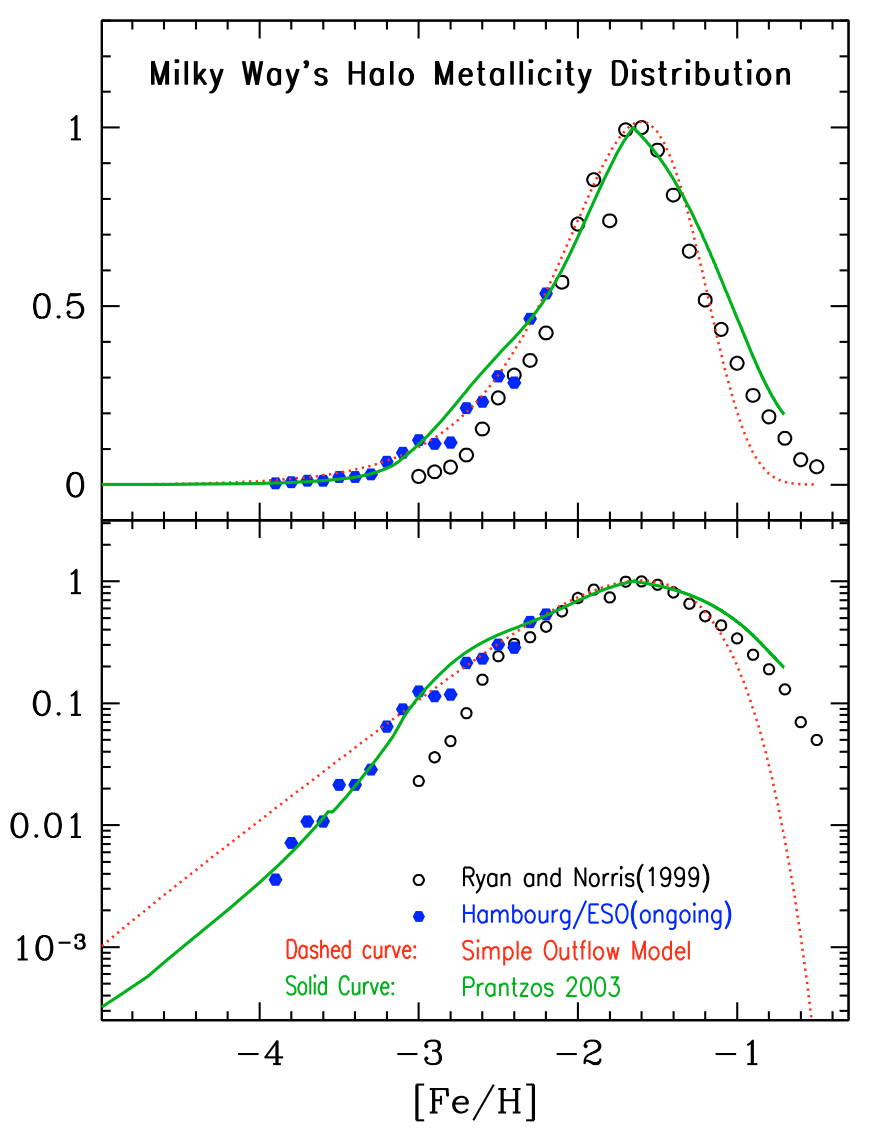

Fig. 1. Differential metallicity distribution of field halo stars in linear (top) and logarithmic (bottom) scales. Data are from Ryan \& Norris (1991, open symbols), and the ongoing Hamburg/ESO project (filled symbols). The two data sets are normalised at $[\mathrm{Fe} / \mathrm{H}]-2.2$; above that value, the Hamburg/ESO data are incomplete, while below that metallicity the Ryan and Norris (1991) data set is incomplete. The dotted curve is a simple model with instantaneous recycling (IRA) and outflow rate equal to 8 times the star formation rate. The solid curve is obtained as in Prantzos (2003), from a model without IRA, an early phase of rapid infall and a constant outflow rate equal to 7 times the SFR. All curves and data are normalised to $\max =1$.

The high metallicity tail of the halo DMD may also hold some important clues as to the halo evolution. Its shape is surprisingly well fitted by the simple model, up to $[\mathrm{Fe} / \mathrm{H}] \sim-1$. In the framework of the simple model, this implies that the halo evolved down to a very low gas fraction $\mu\left(\leq Z_{1}\right) \sim 0.05$. Indeed, since $\log \left(Z_{1}\right) \sim-1$ and $\log \left(p_{\text {Halo }}\right) \sim-1.6$, the main result of the simple model (Eq. (1)) leads to $\mu \sim 0.05$. However, a different interpretation was suggested by Oey (2003). In the framework of her simple inhomogeneous model, the halo is a relatively unevolved system, composed of a number of regions enriched to different levels (the larger ones having lower metallicities). In that model, the final metallicity distribution essentially reflects the size distribution of those regions, and at high metallicities it scales as $Z^{-2}$, in satisfactory agreement with observations.

At present, it is hard to distinguish between the two alternatives, i.e. to decide whether the strong decrease of the observed star number in the high metallicity tail of the halo is due to the fact that the system is highly evolved or to its inhomogeneous nature. Obviously, it cannot be due to observational errors alone, since the width of the DMD $(\sim 1 \mathrm{dex})$ is much larger than the one justified by such errors $(0.1-0.2 \mathrm{dex})$. We simply note that in the modern framework of galaxy formation through hierarchical merging, smaller units have lower metallicities, as discussed in the next section.

\section{The halo DMD and hierarchical merging}

In this work we assume that the MW halo was formed by the merging of smaller units ("sub-haloes"), as implied by the hierarchical merging scenario of galaxy formation. Despite its overall sucess with large scale structure in the Universe, this scenario still faces important problems when it comes to predictions concerning the behaviour of baryons at galactic scales. Among those problems, the one concerning the formation chronology of baryonic vs dark matter structures (namely the fact that large galaxies are observed to be older than small ones, while large dark matter haloes assemble later than low mass ones) is known as downsizing ${ }^{1}$. The word downsizing was first used by Cowie et al. (1996) on observational grounds, while the problem with spirals was noticed in Boissier \& Prantzos (2000) and for ellipticals in Thomas et al. (2002). van den Bosch (2002) showed explicitly how semi-analytical models in the framework of hierarchical merging scenarios fail to reproduce the colour-magnitude relation of galaxies, even if some form of feed-back is included. Despite several attempts, no universally accepted model meeting the observational requirements has been proposed up to now (but see Cattaneo et al. 2008, for a possible solution in the case of elliptical galaxies).

Despite this important shortcoming, several models have been built in the framework of the hierarchical merging scenario, exploring its consequences on the formation of the MW halo and some of them specifically address the issue of the halo metallicity distribution (e.g. Tumlinson 2006; Salvadori et al. 2007, 2008). In these models star formation takes place early on in the different baryonic sub-haloes, which subsequently are tidally disrupted to form a halo component with an old stellar population. Such an early star formation is required to account for the observed age of halo stars (>11 Gyr, e.g. Hill et al. 2002) and their large $[\alpha / \mathrm{Fe}] \sim 0.5$ ratio (Cayrel et al. 2004), suggesting that those stars had not been enriched by Fe from SNIa.

We shall assume then that the MW sub-haloes formed their stars early on, plausibly because at those early times they already evolved in a dense environment (i.e. the deep potential well of the MW halo); similar size sub-haloes in less dense environments should form their stars at a slower pace, on average, and thus develop younger stellar populations today. During the short period of star fomation in each of the sub-haloes, their shallow individual potential wells were unable to keep their gas (affected by ram-pressure stripping and heated by supernovae) which would then leave the sub-haloes (probably to end up in the bulge, as suggested long ago on the basis of angular momentum conservation arguments, e.g. Gilmore et al. 1989). Obviously, the amount of outflowing gas (and of the metals kept inside the system, i.e. the effective yield) should depend on the depth of the potential well of each sub-halo, i.e. on the mass of the corresponding dark matter sub-halo.

In order to calculate semi-analytically the resulting DMD in the framework of the aforementionned scenario, it is further assumed that each of the sub-haloes had a DMD well described by the simple model, i.e. by Eq. (2). It remains then to evaluate the corresponding effective yield $p(M)$ of each sub-halo as a

\footnotetext{
${ }^{1}$ Over the years, the word downsizing has been used to denote several different, albeit not quite unrelated, concepts (see Conroy \& Weschler 2008).
} 
function of the mass of the sub-halo, as well as the mass function of the sub-haloes $\mathrm{d} N / \mathrm{d} M$, in order to obtain the required result. Each one of those ingredients is discussed in the following sections.

\subsection{The shape of sub-halo DMD}

It is assumed here that each of the merging sub-haloes has a DMD described by the simple model with an appropriate effective yield. This assumption is based on recent observations of the dwarf spheroidal (dSph) satellites of the Milky Way, as will be discussed below. It is true that the dSphs that we see today cannot be the components of the MW halo, because of their observed abundance patterns: indeed, as discussed in several places (e.g. Shetrone et al. 2001; Venn et al. 2004) their $\alpha / \mathrm{Fe}$ ratios are typically smaller than the $[\mathrm{a} / \mathrm{Fe}] \sim 0.4 \sim$ const. ratio of halo stars. This implies that they evolved on longer timescales than the Galactic halo, allowing SNIa to enrich their ISM with $\mathrm{Fe}$ peak nuclei and thus to lower the $\alpha / \mathrm{Fe}$ ratio by a factor of $\sim 2$ (as evidenced from the $[\mathrm{O} / \mathrm{Fe}] \sim 0$ ratio in their highest metallicity stars, about half of the MW halo value). However, the shape of the DMD of the simple model does not depend on the star formation history or the evolutionary timescale. This is precisely what makes the DMD such a powerful diagnostic tool: it provides accurate and indepedent information on gas flows into and out of the system, as well as on the initial metallicity. In other terms, the question is whether the components of the MW halo had a DMD decribed by the simple model or by some different form. To answer that question, one may turn either to simulations (which are difficult to constrain) or to observations of galaxian systems smaller than the MW halo. This is the case of the Local Group dSphs.

Helmi et al. (2006) report observations of several hundred red giant stars in four nearby dSphs (Sculptor, Fornax, Sextans and Carina), obtained with the FLAMES spectrograph in the VLT. The surface $[\mathrm{Fe} / \mathrm{H}]$ ratio of those stars should only marginally be affected by the 1 st dredge-up, and thus they may be used as tracers of the chemical evolution of the corresponding galaxies. The resulting DMDs are displayed as histograms in Fig. 2, where they are compared to the simple model with appropriate effective yields (solid curves). The effective yield in each case was simply assumed to equal the peak metallicity (Eq. (2)). It can be seen that the overall shape of the DMDs is quite well fitted by the simple models. This is important, since i) it strongly suggests that all DMDs of small galaxian systems can be described by the simple model (at least, there are no counter examples to that hypothesis) and ii) it allows us to determine effective yields by simply taking the peak metallicity of each DMD; this will be used in Sect. 3.2 below.

Before proceeding to the determination of effective yields, we note that the fit of the simple model to the data of dSphs fails in the low metallicity tails: as already noticed in Helmi et al. (2006), there is a lack of low metallicity stars w.r.t. the simple model predictions. Helmi et al. (2006) attribute this to a preenrichment of the gas out of which the dSphs were formed, i.e. they assume that $Z_{0} \neq 0$ in Eqs. (1) and (2). By fitting their data that way, they determine then initial metallicities in the range $[\mathrm{Fe} / \mathrm{H}]_{0} \sim-2.9--2.7$ for the four galaxies. Furthermore, they argue that this result has a strong implication; it would imply that "... the progenitors of the Milky way (halo) and of dSphs must have been different". However, this is not necessarily true.

Indeed, the idea of a finite initial metallicity (preenrichment) was for a long time considered as the "simplest" solution to the G-dwarf problem in the solar neighborhood (see

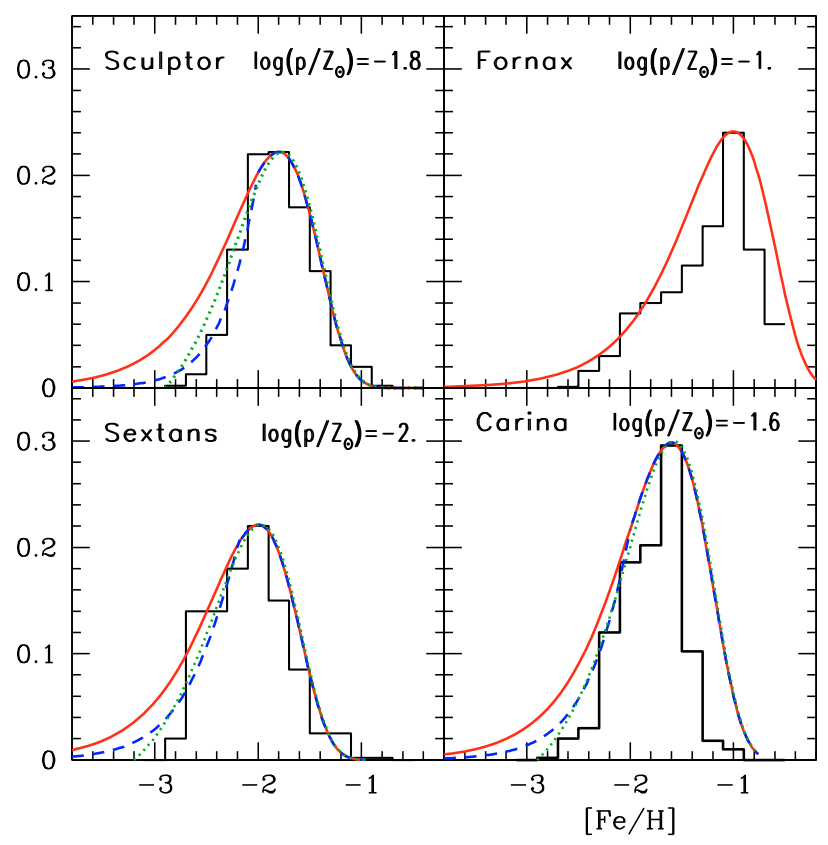

Fig. 2. Metallicity distributions of dwarf satellites of the Milky Way. Data are in histograms (from Helmi et al. 2006). Solid curves indicate the results of simple GCE models with outflow proportional to the star formation rate; the corresponding effective yields (in $Z_{\odot}$ ) appear on top right of each panel. Dashed curves are fits obtained with an early infall phase, while dotted curves are models with an initial metallicity $\log \left(Z_{0}\right) \sim-3$; both modifications of the simple model (i.e. infall and initial metallicity) improve the fits to the data.

Pagel 1997). But, although mathematically the simplest, it was not the most "natural", and infall has now superceded it. In the case of the MW halo, the situation is less clear, but early infall appears again as a possible scenario, as argued in Prantzos (2003) and in Sect. 3.1. In the light of these arguments, one should not exclude that early infall is also the solution to the "low metallicity tail" problem of the DMDs of the local dSphs. If an early infall phase turns out indeed to be a universal feature of galaxian systems, then the MW halo may well be composed of galaxies similar to the progenitors of the dSphs. We shall return to the low metallicity tail of the halo in Sect. 3.5, after considering the bulk of the halo DMD.

\subsection{The effective yield of the sub-haloes}

If the DMDs of all the components of the Galactic halo are described by the simple model, then their shape is essentially described by the corresponding effective yield $p$ (and, to a lesser degree, by the corresponding initial metallicity $Z_{0}$ ). Observations suggest that the effective yield is a monotonically increasing function of the galaxy's stellar mass $M_{*}$. Data for about 40 galaxies of the Local Group, compiled by Dekel and Woo (2003) are presented in Fig. 3. It can be seen that the (median) stellar metallicity of these galaxies varies as $0.01\left(M_{*} / 10^{6} M_{\odot}\right)^{0.4}$. Dekel $\&$ Woo (2003) offer a plausible theoretical framework for that corelation, based on supernova feedback and larger mass loss from smaller galaxies; their model satisfactorily explains other observed properties, such as velocity dispersion and central surface brightness vs. stellar mass. We assume here that the effective yield is equal to the median stellar metallicity (from Eq. (2)). In the case of the progenitor systems of the MW halo, however, 


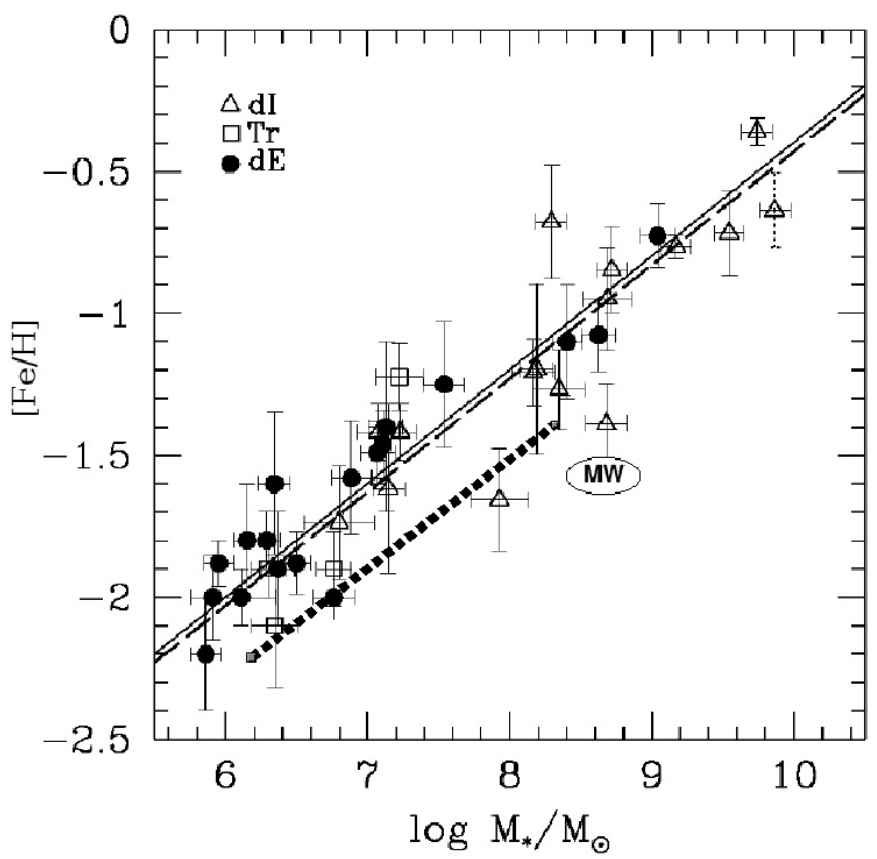

Fig. 3. Stellar metallicity vs stellar mass for nearby galaxies; data and model (upper curves) are from Dekel \& Woo (2003), with $d I$ standing for dwarf irregulars and $d E$ for dwarf ellipticals. The thick dotted line represents the effective yield of the sub-haloes that formed the MW halo according to this work (i.e. with no contribution from SNIa, see Sect. 3.2). The MW halo, with average metallicity $[\mathrm{Fe} / \mathrm{H}]=-1.6$ and estimated mass $\sim 4 \times 10^{8} M_{\odot}$ falls below both curves.

the effective yield must have been lower, since SNIa had no time to contribute (as evidenced by the high $\alpha / \mathrm{Fe} \sim 0.4$ ratios of halo stars), by a factor of about two (see discussion in previous section). We assume then that the effective yield of the MW halo components (accreted satellites or sub-haloes) is given (in solar units $Z_{\odot}$ ) by

$p\left(M_{*}\right)=0.005\left(\frac{M_{*}}{10^{6} M_{\odot}}\right)^{0.4}$

i.e. the thick dotted curve in Fig. 3.

Obviously, the stellar mass $M_{*}$ of each of the sub-haloes should be $M_{*}<M_{\mathrm{H}}$ where $M_{\mathrm{H}}$ is the stellar mass of the MW halo. In a recent paper, Bell et al. (2008), analysing $4 \times 10^{6}$ stars of the MW halo found that the stellar mass in the halo region at distances 1 to $40 \mathrm{kpc}$ from the Galactic center is $M_{\mathrm{H}}=4 \pm 0.8 \times 10^{8} M_{\odot}$ and we adopt that value here. The conclusions of our work depend only slightly on the precise value of $M_{\mathrm{H}}$.

An inspection of Fig. 3 shows that the effective yield of the MW halo is substantially lower (by a factor of $\sim 4$ ) than that of dwarf galaxies with stellar masses in the range of $M_{\mathrm{H}}$. Part of this difference (a factor of $\sim 2$ ) is due to the fact that no SNIa contributed to the effective Fe yield of the MW halo. The remaining difference can be qualitatively understood if it is assumed that the halo was formed by the assembly of smaller mass satellites: the resulting total mass $\left(M_{\mathrm{H}}\right)$ is greater than the mass of the largest sub-halo, but the resulting mass-weighted effective yield $\left(p_{\mathrm{H}}\right)$ is smaller than the effective yield of the largest sub-halo. On the contrary, if the MW halo were formed as a single system, it is hard to understand why its effective yield is so low w.r.t. systems of similar mass.

\subsection{The stellar mass function of the sub-haloes}

Contrary to the cases of the effective yield and of the shape of the DMD, the mass function of the sub-haloes that presumably formed the MW halo cannot be observed today (since the observed number of local dwarf galaxies is insufficient for that purpose) but has to be derived from semi-analytical or numerical calculations of structure formation.

Hierarchical galaxy formation scenarios predict the mass function of the dark matter sub-haloes that compose a dark matter halo at a given redshift. In the high resolution simulation Via Lactea, one of the most "realistic" simulations of a MWlike galaxy performed so far, Diemand et al. (2007) find that the cumulative dark sub-halo mass function at redshift $z=0$ can well be approximated by a power-law $N\left(>M_{\mathrm{D}}\right)=0.0064$ $\left(M_{\mathrm{D}} / M_{\mathrm{DH}}\right)^{-1}\left(M_{\mathrm{D}}\right.$ being the mass of the dark matter sub-haloes and $M_{\mathrm{DH}}$ the total mass of the dark halo); this approximation is valid over three orders of magnitude, i.e. from $3 \times 10^{6}$ to $3 \times 10^{9} M_{\odot}$ inside the galactic virial radius.

The corresponding differential mass function is obviously

$$
\frac{\mathrm{d} N}{\mathrm{~d} M_{\mathrm{D}}} \propto M_{\mathrm{D}}^{-2}
$$

Salvadori et al. (2007) find in their numerical simulations that the same result is valid at higher redshifts (even up to $z=5$, see their Fig. 3), at least for the low mass part of the dark halo mass function. In a recent work, Giocoli et al. (2008) find analytically (by applying the extended Press Schecter formalism and using the progenitor (conditional) mass function) that the result of Eq. (5) is independant of the mass of the progenitor halo and extends down to sub-halo masses as low as $10^{-6}$ of that mass.

However, in our case, we are interested in the mass function of the stellar sub-haloes, and not of the dark ones. The initial ratio of baryonic mass $M_{\mathrm{B}}$ to dark matter mass is usually taken to be independent of the dark halo mass $M_{\mathrm{D}}$ and equal to the corresponding cosmic ratio $f=\Omega_{\mathrm{B}} / \Omega_{\mathrm{D}} \sim 0.17$, where $\Omega_{\mathrm{B}} \sim 0.04$ and $\Omega_{\mathrm{D}} \sim 0.23$ are the cosmic densities of baryons and dark matter, respectively (e.g. Fukugita \& Peebles 2003). If that ratio remained constant throughout galaxy evolution, then Eq. (5) would obviously provide also the required stellar mass function (assuming that all, or most, baryons form stars). However, several effects may subsequently alter that ratio, e.g. ram pressure stripping or galactic winds from supernova feedback. Observations and theoretical arguments suggest that those effects become more important in lower mass systems (e.g. Dekel \& Woo 2003). It is difficult to evaluate the impact of those effects on the final baryonic fraction of a given galaxy, and to derive the corresponding mass function of baryonic (mostly stellar) sub-haloes from first principles. We attempt here an analytical evaluation of those quantities, based on the various empirical relations established in Sects. 2 and 3.

From Eq. (3), one can see that the ratio of the outflow to the star formation rate from a galaxy (or a sub-halo) of stellar mass $M_{*}$ is

$k\left(M_{*}\right) \propto p\left(M_{*}\right)^{-1}$

since $p_{\text {True }} \gg p\left(M_{*}\right)$ for those small galaxies/sub-haloes, which are dominated by outflows (as can be seen in Fig. 4, top panel, even for a galaxy of $M_{*} \sim 10^{8} M_{\odot}$, the outflow rate is 3 times the SFR). By virtue of Eq. (4), one has then $k\left(M_{*}\right) \propto M_{*}^{-0.4}$, i.e.

$\frac{M_{\mathrm{OUT}}}{M_{*}} \propto M_{*}^{-0.4}$ 


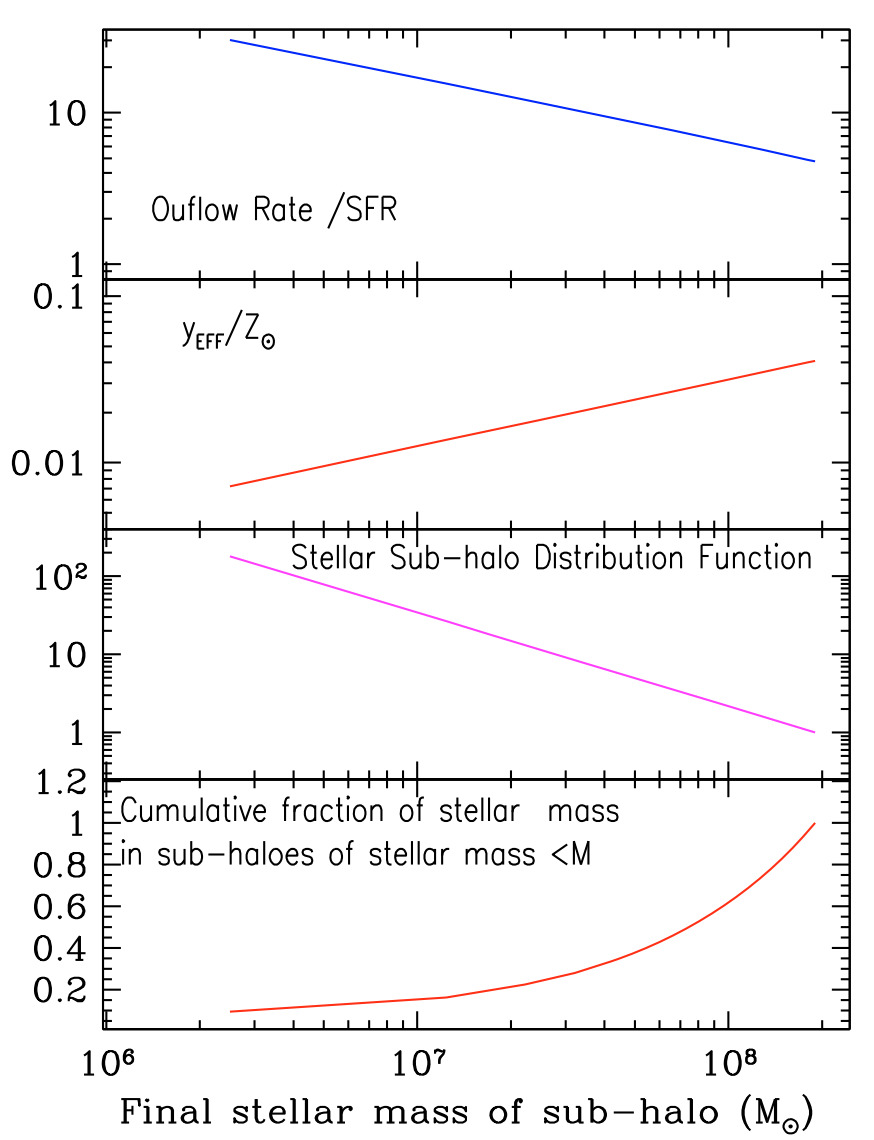

Fig. 4. Properties of the sub-haloes as a function of their stellar mass, empirically derived as discussed in Sect. 3. From top to bottom: Outflow rate, in units of the corresponding star formation rate; effective yield, in solar units; distribution function; cumulative fraction of stellar mass contributed by the sub-haloes. The total mass of the MW halo is $4 \times 10^{8} M_{\odot}$.

where $M_{\text {OUT }}=\int F \mathrm{~d} t=\int k \Psi \mathrm{d} t$ is the mass lost from the system and $M_{*}=\int \Psi \mathrm{d} t$ is the stellar mass of the system. On the other hand, the baryonic mass $M_{\mathrm{B}}$ is given by

$M_{\mathrm{OUT}}+M_{*}=M_{\mathrm{B}}=f M_{\mathrm{D}}$

with $f=\Omega_{\mathrm{B}} / \Omega_{\mathrm{D}}$.

For $M_{\mathrm{OUT}} \gg M_{*}$, Eqs. (7) and (8) lead to $M_{*}^{0.6} \propto M_{\mathrm{D}}$. Combining this with the distribution function of the dark matter sub-haloes (Eq. (5)), one finally gets

$\frac{\mathrm{d} N}{\mathrm{~d} M_{*}} \propto M_{*}^{-1.2}$

i.e. the distribution function of the stellar sub-haloes is flatter than the distribution function of the dark matter sub-haloes because of the mass loss, which becomes progressively stronger as we move to less massive sub-haloes. The normalisation of the stellar sub-halo distribution function is made through

$\int_{M_{1}}^{M_{2}} \frac{\mathrm{d} N}{\mathrm{~d} M_{*}} M_{*} \mathrm{~d} M_{*}=M_{\mathrm{H}}$

where $M_{\mathrm{H}}=4 \times 10^{8} M_{\odot}$ is the adopted stellar mass of the MW halo.

The lower mass limit $M_{1}$ is adopted here to be $M_{1}=1-2 \times$ $10^{6} M_{\odot}$, in agreement with the lower mass bound of observed
dSphs in the Local group (see Fig. 3). Such galaxies have internal velocities $V>10 \mathrm{~km} \mathrm{~s}^{-1}$. Dekel \& Woo (2003) argue that the gas in haloes with $V<10 \mathrm{~km} \mathrm{~s}^{-1}$ cannot cool to form stars at any early epoch and that dwarf ellipticals form in haloes with $10<V<30 \mathrm{~km} \mathrm{~s}^{-1}$. From their $V$ vs. $M_{*}$ diagrams (their Figs. 3 and 5) one sees that a velocity of $30 \mathrm{~km} \mathrm{~s}^{-1}$ corresponds to a stellar mass $M_{*} \sim 2 \times 10^{8} M_{\odot}$, i.e. about half the adopted stellar mass of the MW halo. This is the highest conceivable mass for a component of a $\sim 4 \times 10^{8} M_{\odot}$ halo and it is the upper mass limit $M_{2}$ that we adopt here for Eq. (10).

The main properties of the sub-halo set constructed in this section appear in Fig. 4 as a function of the stellar sub-halo mass $M_{*}$.

\subsection{The composite stellar halo DMD}

We now have all the ingredients required to calculate the DMD of the MW halo, assuming that

1) It is composed of a number of sub-haloes with stellar masses ranging from $M_{1}=2 \times 10^{6} M_{\odot}$ to $M_{2}=2 \times 10^{8} M_{\odot}$ and distributed as in Eq. (9); and

2) Each of the stellar sub-haloes has a DMD given by the simple model (Eq. (2)) with a mass dependent effective yield given by Eq. (4).

The resulting DMD is then obtained as a sum over all subhaloes:

$$
\frac{\mathrm{d}\left(n / n_{1}\right)}{\mathrm{d}(\log Z)}=\int_{M_{1}}^{M_{2}} \frac{\mathrm{d}\left[n\left(M_{*}\right) / n_{1}\left(M_{*}\right)\right]}{\mathrm{d}(\log Z)} \frac{\mathrm{d} N}{\mathrm{~d} M_{*}} M_{*} \mathrm{~d} M_{*} .
$$

The result appears in Fig. 5 (top panel on linear and middle panel on logarithmic scales, respectively). It can be seen that it fits the observed DMDs at least as well as the simple model of Hartwick (1976). In fact, at low metallicities, the behaviour of the simple model (with outflow rate $=8$ times the SFR) is similar to the one of the composite model. The reason is that far from the peak (given by the effective yield) the DMDs of all individual haloes have the same slope (see middle panel of Fig. 5) and so does their sum. In the high metallicity region, the composite model performs slightly better than the simple model. This region is dominated by the DMDs of the most massive haloes, which peak at $[\mathrm{Fe} / \mathrm{H}] \sim-1$, whereas at low metallicities the small haloes dominate the composite DMD (see bottom panel in Fig. 5).

In summary, under the assumptions made in this section, the bulk of the DMD of the MW halo can be obtained as the sum of the DMDs of the component sub-haloes. All the ingredients of the analytical model are taken from observations of local satellite galaxies, except for the adopted mass function of the sub-haloes (which results from the analytical theory of structure formation plus a small modification to account for the role of outflows). Obviously, by assuming different values for the slope of the dark matter haloes in Eq. (5) and for the mass limits $M_{1}$ and $M_{2}$ in Eqs. (10) and (11), one may modify the peak of the resulting composite DMD, thus allowing for differences between the halo DMDs of different galaxies.

\subsection{The low metallicity tail of the DMD}

As already discussed in Sect. 2, current surveys of the low metallicity tail of the halo DMD find a lack of stars w.r.t. the predictions of the simple model (with outflow) below $[\mathrm{Fe} / \mathrm{H}] \sim-3$.

\footnotetext{
${ }^{2}$ Dekel \& Woo (2003) calculate internal velocity as $V=\sqrt{3} \sigma_{\mathrm{p}}$, where $\sigma_{\mathrm{p}}$ is the projected central velocity dispersion.
} 


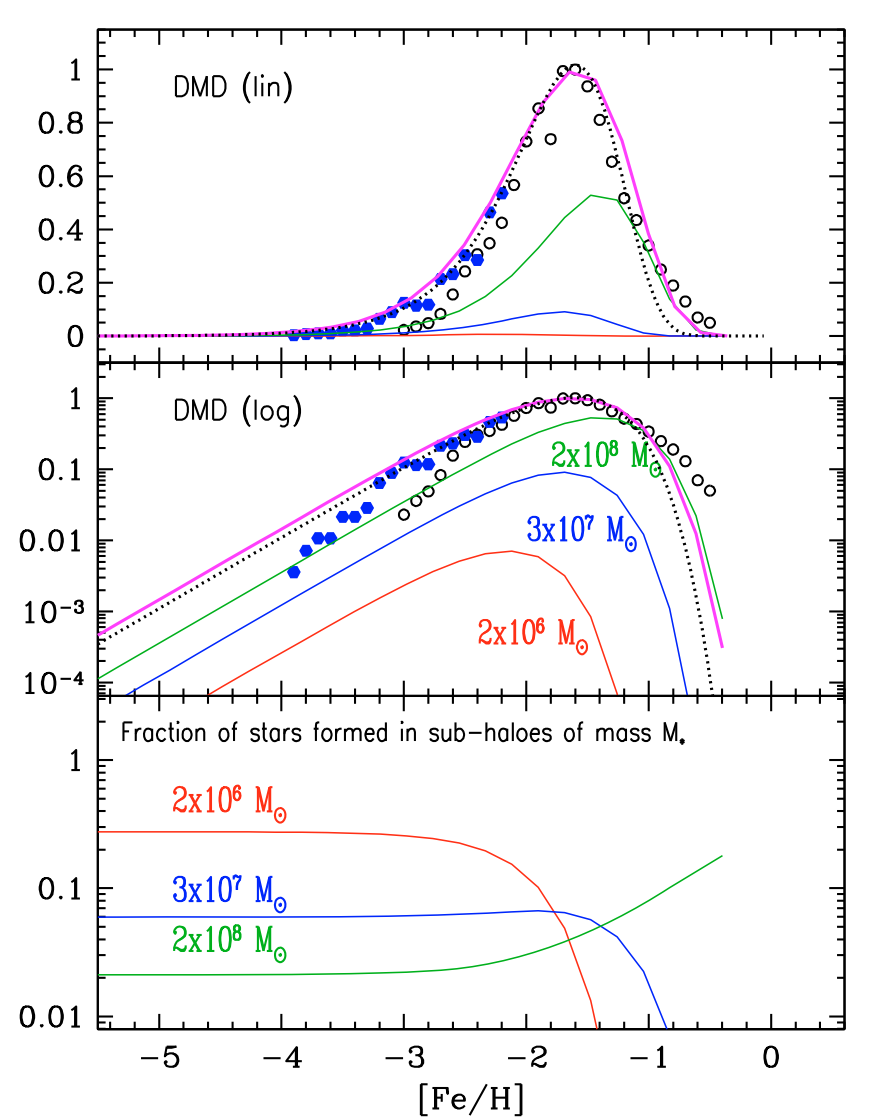

Fig. 5. Top and middle panels: differential metallicity distribution (on linear and logarithmic scales, respectively) of the MW halo, assumed to be composed of a population of smaller units (sub-haloes). The individual DMDs of a few sub-haloes, from $10^{6} M_{\odot}$ to $4 \times 10^{7} M_{\odot}$, are indicated in the middle panel, as well as the sum over all haloes (solid upper curves in both panels, compared to observations). Dotted curves in top and middle panels indicate the results of the simple model with outflow (same as in Fig. 1). Because of their large number, small subhaloes with low effective yields contribute the largest fraction of the lowest metallicity stars, while large haloes contribute most of the high metallicity stars (bottom panel).

A similar trend is found in satellite galaxies of the Milky Way by Helmi et al. (2006), albeit at slightly higher metallicities $([\mathrm{Fe} / \mathrm{H}] \sim-2.8)$. In the case of the MW halo, Prantzos (2003) argued that an early infall phase may account for the discrepancy (see Fig. 1). Obviously, the same solution may also apply to the case of the MW satellites, i.e. the conclusion of Helmi et al. (2006) that those galaxies were formed from pre-enriched gas (of $[\mathrm{Fe} / \mathrm{H}] \sim-2.8$ ) does not necessarily hold. Consequently, the main point of Helmi et al. (2006), namely that the MW halo was not formed from sub-haloes similar to the currently observed satellites, may not hold either. Indeed, if an early infall phase is assumed for all the constituent sub-haloes, the composite halo DMD will necesarily display a deficit of low metallicity stars, in agreement with the observations. However, it is impossible at present to determine the characteristics of the infall for each one of the sub-haloes as a function of their mass.

For illustration purposes, we adopt here a simple model, where each sub-halo undergoes an early infall phase (of primordial composition), with the smaller sub-haloes having stronger infall. This prescription for infall is motivated from the observed

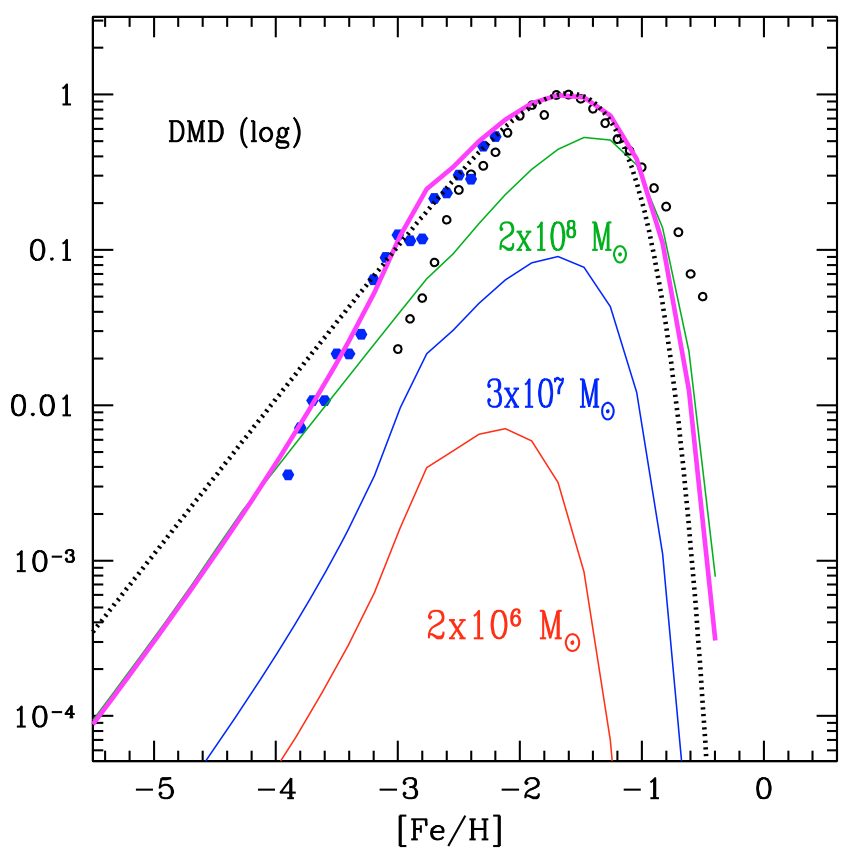

Fig. 6. Differential metallicity distribution of the composite MW halo population. The difference with Fig. 5 is that an early infall phase is assumed for each one of the component sub-haloes (except the most massive ones, see Sect. 3.5), so as to produce a paucity of low metallicity stars. The dotted curve is the simple outflow model; data is as in Fig. 1.

DMDs of the satellite dSphs of the MW: as can be seen in Fig. 2, the most massive satellites, like Fornax, are better described by the simple outflow model, whereas in the low mass satellites the simple model fails in the low metallicity tail and requires strong early infall. In all cases, outflow at an appropriate rate (reproducing the observed effective yield) is assumed.

The results of the model appear in Fig. 6. Because of the assumed early infall, the low metallicity tails of the small subhaloes are less pronounced than in the simple model. Since those sub-haloes dominate the local DMD (because of their large number and low metallicity peaks), the low metallicity tail of the composite DMD is also affected: the corresponding curve now fits the (preliminary) Hamburg/ESO survey data. Thus, it is possible that all types of galaxian systems, namely both the components of the MW halo and the small dSphs of the local group, have undergone an early period of infall, shaping the low metallicity tails of their DMDs.

\section{Summary}

In this work we present an analytical model for the metallicity distribution of the MW halo, in the framework of the hierarchical merging scenario for galaxy formation. It is assumed that the MW halo was composed of a number of sub-haloes with stellar masses ranging from $M_{1}=2 \times 10^{6} M_{\odot}$ to $M_{2}=2 \times 10^{8} M_{\odot}$ and distributed as in Eq. (9). Furthermore, each of the stellar subhaloes has a DMD given by the simple model (Eq. (2)) with a mass-dependent effective yield given by Eq. (4). These assumptions are based on observations of dSph satellites of the MW galaxy, while the distribution function is obtained from numerical simulations of dark matter halo formation (after explicitly accounting for modification due to outflows). 
It is found that, under those assumptions, the bulk of the MW halo DMD is reproduced in a fairly satisfactory way. The advantage of that model over the simple outflow model (proposed by Hartwick in 1976) is that it treats the MW halo not as a single entity but as a composite one, in line with the hierarchical merging scenario for galaxy formation. Furthermore, the proposed composite model avoids the "paradox" noticed in Sect. 3.2, namely that the effective yield of the MW halo appears to be substantially lower than the effective yields of dwarf galaxies of comparable mass.

Observations suggest that the low metallicity tail of both the MW halo and local dSphs is deficient in stars compared to the predictions of the simple outflow model. Prantzos (2003) suggested that early infall may account for that defficiency in the case of the MW halo, while Helmi et al. (2006) prefer preenrichment (at a level of $[\mathrm{Fe} / \mathrm{H}]=\sim-2.8$ ) for the dSphs. It is shown here, with a simple model based on observations of dSphs, that an early infall phase can account for the low metallicity tails of all small galaxian systems and, consequently, of the MW halo conceived as a composite system.

The results obtained here with semi-analytical models should be substantiated with detailed numerical simulations of the formation of a stellar halo in a MW-type galaxy. Such a simulation was recently performed by Salvadori et al. (2008), who also investigated the formation and evolution of dSphs along the hierarchical build-up of the MW, matching several observed properties of those galaxian systems. In their scenario, dSphs are associated to Galactic progenitors correponding to low-sigma density fluctuations, that virialize out of the MW environment before the end of reionization (at redshift $z \sim 7$ ). At that epoch the MW environment is metal-enriched (due to previous SN explosions), up to $[\mathrm{Fe} / \mathrm{H}] \sim-3$, a value comparable to that inferred from the observations by Helmi et al. (2006). Note, however, that the typical dSph model of Salvadori et al. (2008) undergoes also an early infall phase lasting for $\sim 0.1$ Gyr (their Fig. 2), which should also affect the shape of the resulting DMD. Thus, the issue of pre-enrichment vs. early infall for the MW satellites is not settled yet and it may well be that both factors contribute to the DMD of local dSphs. The simulations of Salvadori et al. (2008) provide support to the analytical scenario presented here, and offer a global and satisfactory picture of the formation of the halo and the satellites of the MW. It should be noticed, however, that such simulations still suffer from several drawbacks, as also acknowledged by the authors themselves.

Finally, as emphasized in Sect. 2, an in-depth discussion of the important implications of the low metallicity tail of the DMD should be based on the final release of the results of the
Hamburg/ESO survey (Schörck et al., in preparation); the model presented here relies on preliminary results and only illustrates the potential of the proposed analytical method to probe the physics of the early galaxy.

\section{References}

Bekki, K., \& Chiba, M. 2001, ApJ, 558, 666

Bell, E., \& Zuker, D, Belokurov, V. et al., 2008, ApJ 680, 295

Boissier, S., \& Prantzos, N. 1999, MNRAS, 307, 857

Boissier, S., \& Prantzos, N. 2000, MNRAS, 312, 398

Carney, B., Laird, J., Latham, D., \& Aguilar, L. 1996, AJ, 112, 668

Cattaneo, A., Dekel, A., Faber, S. M., \& Guiderdoni, B. 2008 [arXiv: 0801.1673]

Cayrel, R., Depagne, E., Spite, M., et al. 2004, A\&A, 416, 1117

Conroy, C., \& Wechsler, R. 2008 [arXiv: 0805.3346]

Cowie, L., Songaila, A., Hu, E. M. \& Cohen, J. G. 1996, AJ, 112, 839

Dekel, A., \& Woo, J. 2003, MNRAS, 344, 1131

Diemand, J., Kuhlen, M., \& Madau, P. 2007, ApJ, 667, 859

Edmunds, M. 1990, MNRAS 246, 678

Eggen, O., Lynden-Bell, D., \& Sandage, A. 1962, ApJ, 136, 748

Font, A., Johnston, K., Bullock, J., \& Robertson, B. 2006, ApJ, 638, 585

Fukugita, M., \& Peebles, P. J. E. 2004, ApJ, 616, 643

Fulbright, J., McWilliam, A., \& Rich, M. 2006, ApJ, 636, 821

Gilmore, G., Wyse, R., \& Kuijken, K. 1989, ARA\& A, 27, 555

Giocoli, C., Pieri, L., \& Tormen, G. 2008, MNRAS, 387, 689

Goswami, A., \& Prantzos, N. 2000, A\&A, 359, 151

Hartwick, F. 1976, ApJ, 209, 418

Haywood, M. 2006, MNRAS, 371, 176

Helmi, A., Irwin, M., Tolstoy, E., et al. 2006, ApJ, 651, L121

Hill, V., Plez, B., Cayrel, R., et al. 2002, A\&A, 387, 560

Holmberg, J., Nordström, B., \& Andersen, J. 2007, A\&A, 475, 519

Kroupa, P. 2002, Science 295, 82

Kroupa, P., Tout, C., \& Gilmore, G. 1993, MNRAS, 262, 545

Oey, K. 2003, MNRAS 339, 849

Pagel, B. 1997, Nucleosynthesis and galactic chemical evolution (Cambridge University Press)

Prantzos, N. 2003, A\&A, 404, 211

Prantzos, N. 2007a, in Stellar Nucleosynthesis: 50 years after B2FH, ed. C. Charbonnel \& J. P. Zahn, EAS Publ. Ser. [arXiv:0709.0833]

Prantzos, N. 2007b, in CRAL-2006: Chemodynamics, from first stars to local galaxies, ed. E. Emsellem et al., EAS Publ. Ser., 24, 3

Norris, J. 1999, in The Galactic Halo, ed. B. Gibson, T. Axelrod \& M. Putnam, ASP Conf. Ser., 165, 213

Ryan, S., \& Norris, J. 1991, AJ, 101, 1865

Salvadori, S, Schneider, R., \& Ferrara, A. 2007, MNRAS, 381, 647

Salvadori, S., Ferrara, A., \& Schneider, R. 2008, MNRAS, 386, 348

Scanapieco, E., \& Broadhurst, T. 2001, ApJ, 550, L39

Shetrone, M., Cote, P., \& Sargent, W. 2001, ApJ, 548, 592

Thomas, D., Maraston, C., \& Bender, R. 2002, Ap\&SS, 281, 371

Tsujimoto, T., Shigeyama, T., \& Yoshii Y. 1999, ApJ 519, L63

Tumlinson, J. 2006, ApJ, 641, 1

van den Bosch, F. 2002, MNRAS, 332, 456

Venn, K., Irwin, M., Shetrone, M., et al. 2004, ApJ, 128, 1177 